\title{
Surface Contribution to Thermodynamic Properties of Solids
}

\author{
P.N. Ram ${ }^{a, *}$, M. Kushwaha ${ }^{b}$ And A. Kumar Chatterjee ${ }^{b}$ \\ ${ }^{a}$ Department of Chemical Engineering, M.J.P. Rohilkhand University, \\ Bareilly, Uttar Pradesh, India \\ ${ }^{b}$ Electronics and Communication Engineering Department, \\ Thapar Institute of Engineering and Technology, Patiala, Punjab, India
}

Received: 05.06.2020 \& Accepted: 21.09.2020

Doi: 10.12693/APhysPolA.139.25

*e-mail: pnr1346@rediffmail.com

\begin{abstract}
We present an approach based on local representation of frequency spectrum to calculate the surface contribution to thermodynamic properties of solids. The Green function theory of isolated point defects is discussed in detail which is also applicable to vibrations of surface atoms. The expression for local spectra of atoms is obtained in terms of the same site Green functions. The surface contribution to thermodynamic properties of solids is expressed in terms of the local frequency spectra of atoms in a few surface layers and the frequency spectrum of the infinite crystal satisfying the cyclic boundary condition. The usefulness of the formulation along with the ability of the recursion method to calculate local spectra without a recourse to detailed frequency calculations of slabs is emphasized. As an illustrative example, we present the surface contribution to low temperature lattice specific heat of tungsten due to (100) surface.
\end{abstract}

topics: local density of states, thermodynamic properties, frequency spectrum, lattice specific heat

\section{Introduction}

Generally, the vibrational properties of crystals are discussed on the assumption that the displacements of atoms follow the cyclic or periodic boundary condition of Born and von Karman. Further, it is assumed that by the use of these unphysical boundary conditions, a negligible error is introduced in the calculation of the frequency spectrum and the vibrational contribution to extensive properties of the crystal, since for any bulk sample the number of degrees of freedom is essentially infinite. However, the atoms near a free surface of a real crystal are subjected to different forces than the atoms in the bulk since an atom in the surface layers has fewer neighbours when compared to an atom in the interior of a crystal. As a consequence, the vibrational properties of surface atoms are different from those of the bulk atoms. For finite crystals, when the crystal size is small enough, the ratio of the surface area to the volume of the crystal is not negligible and therefore the modification in the frequency spectrum due to free surfaces must be taken into account. This modification involves an additional contribution from an essentially two-dimensional crystal. The modification in the frequency spectrum due to the surface alters the temperature dependence of the thermodynamic functions of the crystal. For instance, the contribution to low temperature lattice specific heat due to surfaces varies as $T^{2}$ rather than the usual $T^{3}$ dependence for the cyclic crystal. This is because in the limit of low frequencies, the frequency spectrum for a two-dimensional lattice is expected to be proportional to $\omega$ rather than $\omega^{2}$ dependence for the three-dimensional crystal.

Most of the earlier attempts to evaluate surface contribution to thermodynamic functions of solids concern the lattice specific heat. The earliest attempts were based on the elasticity theory $[1,2]$. Notable among the elastic calculations is the one by Dupuis et al. in 1963 [3] who considered a slab of an isotropic elastic solid bounded by two free surfaces perpendicular to $z$-axis. However, these authors did not calculate the normal mode frequencies and the frequency spectrum of the slab. Instead, they replaced the sum over normal mode frequencies by a contour integral and obtained a remarkable simple expression for the surface contribution to lattice specific heat

$$
c_{s}(T)=3 \pi S \xi(3) \frac{k_{\mathrm{B}}^{3} T^{2}}{\hbar^{2}} \frac{2 c_{t}^{4}-3 c_{t}^{2} c_{l}^{2}+3 c_{l}^{4}}{c_{t}^{2} c_{l}^{2}\left(c_{l}^{2}-c_{t}^{2}\right)}
$$

where $k_{\mathrm{B}}$ is the Boltzmann constant, $c_{l}$ and $c_{t}$ are the speed of the sound for longitudinal and transverse waves, $S$ is the surface area of the crystal and $\xi(3)$ is the Riemann zeta function. From some simple lattice models of isotropic solids, analytic results have been obtained [4] which have a strong resemblance with (1). The only case which has been discussed analytically is that of crystals with hexagonal symmetry [5]. The lattice dynamical 
calculations concerning physically realistic models of crystals are purely numerical in nature where a semi-infinite slab consisting of a comparatively small number of atomic layers is considered [6]. In the lattice dynamical approach, the normal mode frequencies are obtained for the slab-shaped crystal by solving the appropriate eigenvalue equation and thermodynamic functions are obtained by direct summation over frequencies. The programme has been implemented by a number of researchers to calculate the surface contribution to lattice specific heat $[7,8]$. The main difficulty in this approach is the smallness of the slab. As it is, at lower temperatures the dominant contribution to specific heat comes from phonons with longer wavelengths so that a situation arises where the wavelength of the phonons is long enough to be comparable to the dimensions of the slab, putting a serious question mark over the validity of the calculation. The limitation of the lattice dynamical approach based on a semi-infinite slab has been discussed by Portz and Maradudin [9] who have used the elasticity theory to calculate the surface contribution to the lowtemperature specific heat of a cubic crystal.

In the present paper, we follow an alternative approach based on local representation of the frequency spectrum of a crystal. The surface contribution to the thermodynamic function of a solid is expressed in terms of the local frequency spectra of atoms in the few surface layers and the frequency spectrum of the infinite crystal satisfying the cyclic boundary condition. This is similar to the calculation of changes in thermodynamic properties due to defects in terms of local frequency spectra of the defect and a few of its neighbors [10]. The local spectra of surface atoms may be obtained in terms of the Green function of a crystal slab $[11,12]$. However, this type of exact calculation of the Green function in the case of a semi-infinite slab would be difficult in complicated cases such as high index surfaces with stepped structures [13] or even in an approximate slab calculation (see e.g. [14]). In fact, the latter slab calculations $[15,16]$ take much thicker slabs but the problem of hybridization between surface states of the two surfaces of the slab remains for the vanishing wave vectors when the wavelength of the phonon is comparable to the thickness of the slab [15]. On the other hand, the local frequency spectra of surface atoms can also be obtained by the method based on continued fractions - the recursion method [17] — in which this problem of hybridization does not arise. The recursion method was successfully used to study the vibration of surface atoms in metals [13, 18-22] and in silicon nanostructures [23]. In this case, spectra are determined by local environment of a particular atom where the choice of a big cluster is not absolutely necessary [18]. In fact, the calculation of normal mode frequencies of the chosen cluster of atoms is avoided in this method. The local density of states (LDS) of an atom is directly calculated as a continued fraction in which the dominant effect is that of the self-interaction and interactions with the neighboring first shell of atoms. The effect of next shells of atoms is relatively smaller and smaller. Thus, the difficulty with summing over long wavelength phonons in the calculation of surface contribution to lattice specific heat within the slab method is conveniently avoided with LDS calculated with the help of the recursion method. The calculated result for lattice specific heat shows the proper behaviour as shown by a calculation based on elasticity theory, as well as lattice dynamical calculations of a slab-shaped crystal [1, 2, 6-8].

In Sect. 2, we present theory to calculate surface contribution to thermodynamic properties of solids using LDS. In Sect. 3, we calculate surface contribution to lattice specific heat of tungsten using calculated LDS of atoms in the first three layers of (100) surface as an illustrative example.

\section{Theory}

In the harmonic approximation, the thermodynamic quantities are represented by additive functions of the normal mode frequencies and, as a result, they can be expressed as averages over the frequency spectrum

$$
X(T)=\sum_{k} x\left(\omega_{k}, T\right)=\int \mathrm{d} \omega x(\omega, T) Z(\omega),
$$

where $x\left(\omega_{k}, T\right)$ is the contribution to the thermodynamic function at temperature $T$ due to a single oscillator of frequency $\omega_{k}$ and $Z(\omega)$ is the frequency spectrum of the crystal. The surface contribution to any thermodynamic property of a finite crystal is given by

$$
\Delta X(T)=\int_{0}^{\infty} \mathrm{d} \omega x(\omega, T)\left(Z(\omega)-Z^{0}(\omega)\right),
$$

where $Z(\omega)$ and $Z^{0}(\omega)$ are the frequency spectra of a finite crystal and the perfect crystal with the cyclic boundary condition, respectively. To evaluate $\Delta X(T)$, one can take a sufficiently thick slab and find out its frequencies and then the frequencies of a perfect crystal and then generate the frequency spectra $Z(\omega)$ and $Z^{0}(\omega)$. In view of the limitations of the slab method, as discussed earlier, we follow an alternative method based on a local representation of the frequency spectrum. The local frequency spectrum or LDS can be expressed in terms of the Green function of the lattice (see Sect. 2.1).

The LDS approach has been widely used to study the vibrations of defects in metals [24-27] and in ionic crystals [28, 29]. This approach is found to be extremely useful for evaluating the formation entropy of vacancies [30, 31]. As far as the surface properties are concerned, Treglia and Deszonqueres [20] have discussed thermodynamical properties for low index surfaces in fcc metals. Using the recursion method, Tang and Aluru [23] have 
presented results for thermodynamical properties for different atom positions in a silicon nanowire. In an interesting development, Iskandar et al. [32] have used measured heat capacities of bare surfacestructured silicon nanostructures to derive a phonon spectrum showing a significant modification in the bulk phonon spectrum. It is considered to be experimental evidence for changes in the phonon spectrum. This result is corroborated by numerous calculations already cited [13, 18-23].

\subsection{Green's function and density of states}

The standard Green function theory for isolated point defects is well developed $[4,10,24]$. As indicated earlier, the formal structure of the theory for point defects and surface atoms is the same as far as LDS is concerned.

For a monoatomic lattice in the harmonic approximation, the equation of motion of an atom in the direction $\alpha$ at site $l$ is given by

$$
M_{l} \ddot{u}_{\alpha}(l, t)+\sum_{l^{\prime} \beta} \Phi_{\alpha \beta}\left(l, l^{\prime}\right) u_{\beta}\left(l^{\prime}, t\right)=0,
$$

where $M_{l}$ is the mass and $u_{\alpha}(l, t)$ is the displacement of the atom. The $\Phi_{\alpha \beta}\left(l, l^{\prime}\right)$ is the $\alpha \beta$ element of the force constants between atoms $l$ and $l^{\prime}$. In terms of a dynamical matrix

$$
D_{\alpha \beta}\left(l, l^{\prime}\right)=\frac{1}{\sqrt{M_{l}}} \Phi_{\alpha \beta}\left(l, l^{\prime}\right) \frac{1}{\sqrt{M_{l^{\prime}}}},
$$

the equation of motion (4) becomes

$\sum_{l^{\prime} \beta} \sqrt{M_{l}}\left(D_{\alpha \beta}\left(l l^{\prime}\right)-\omega^{2} \delta_{\alpha \beta} \delta_{l l^{\prime}}\right) \sqrt{M_{l^{\prime}}} u_{\beta}\left(l^{\prime}\right)=0$

or in the matrix form

$$
\boldsymbol{L}\left(\omega^{2}\right) \boldsymbol{u}=\boldsymbol{M}^{1 / 2}\left(\boldsymbol{D}-\omega^{2} \boldsymbol{I}\right) \boldsymbol{M}^{1 / 2} \boldsymbol{u}=0 .
$$

The Green function is defined as the inverse of the matrix $\boldsymbol{L}\left(\omega^{2}\right)$ :

$$
\boldsymbol{G}(\omega)=\left[\boldsymbol{L}\left(\omega^{2}\right)\right]^{-1}
$$

or

$$
G_{\alpha \beta}\left(l, l^{\prime}\right)=\sqrt{\frac{1}{M_{l} M_{l^{\prime}}}} \sum_{s} \frac{U_{\alpha}^{*}(l, s) U_{\beta}\left(l^{\prime}, s\right)}{\omega_{s}^{2}-(\omega+\mathrm{i} \epsilon)^{2}},
$$

where $U_{\alpha}(l, s)$ is the element of a unitary matrix that diagonalises the dynamical matrix $\mathbf{D}$. The infinitesimal positive quantity $\epsilon$ in (9) indicates that the retarded Green functions are used. The eigenvectors $\boldsymbol{U}(s)$ satisfy the usual orthogonality and closure relations. In the case of the ideal lattice, the eigenvectors $\boldsymbol{U}(s)$ can be chosen to be plane waves since force constants $\Phi\left(l, l^{\prime}\right)$ have a translational symmetry and all masses are equal. Thus

$$
U_{\alpha}(l, \boldsymbol{k} \sigma)=\sqrt{\frac{1}{N}} \boldsymbol{e}_{\alpha}(\boldsymbol{k}, \sigma) \exp (\mathrm{i} \boldsymbol{k} \cdot \boldsymbol{R}(l)),
$$

where $\boldsymbol{k}$ is the wave vector, $\sigma$ is the polarization index, $\boldsymbol{e}_{\alpha}(\boldsymbol{k}, \sigma)$ is the eigenvector of the dynamical matrix belonging to normal mode $(\boldsymbol{k}, \sigma)$ and $N$ is the number of atoms in the lattice. Thus, the ideal lattice Green function is given by

$$
\begin{aligned}
& G_{\alpha \beta}^{0}\left(l, l^{\prime}, \omega\right)=\frac{1}{M^{0} N} \sum_{\boldsymbol{k} \sigma} \frac{\boldsymbol{e}_{\alpha}^{*}(\boldsymbol{k}, \sigma) \boldsymbol{e}_{\beta}(\boldsymbol{k}, \sigma)}{\omega_{\boldsymbol{k} \sigma}^{2}-(\omega+\mathrm{i} \epsilon)^{2}} \\
& \quad \times \exp \left(\mathrm{i} \boldsymbol{k} \cdot\left(\boldsymbol{R}(l)-\boldsymbol{R}\left(l^{\prime}\right)\right)\right),
\end{aligned}
$$

where $\omega_{\boldsymbol{k} \sigma}$ are the eigenfrequencies. In the ideal lattice, $\Phi$ is real and symmetric and therefore the polarization vectors can be chosen as real so that $U^{*}(\boldsymbol{k})=U(-\boldsymbol{k})$ and $\omega_{\boldsymbol{k} \sigma}=\omega_{-\boldsymbol{k} \sigma}$. The exponential in (11) can be replaced by $\cos \left(\boldsymbol{k} \cdot \boldsymbol{R}\left(l-l^{\prime}\right)\right)$ and

$$
\begin{aligned}
& G_{\alpha \beta}^{0}\left(l, l^{\prime}, \omega\right)=G_{\alpha \beta}^{0}\left(\left|l-l^{\prime}\right|, \omega\right)= \\
& G_{\beta \alpha}^{0}\left(\left|l-l^{\prime}\right|, \omega\right) .
\end{aligned}
$$

Using the identity $(x-\mathrm{i} \epsilon)^{-1}=P / x+\mathrm{i} \pi \delta(x)$, the real and imaginary parts of the Green function are

$$
\begin{aligned}
& \operatorname{Re}\left(G_{\alpha \beta}\left(l, l^{\prime}\right)\right)=\operatorname{Re}\left(G_{\alpha \beta}\left(l, l^{\prime},-\omega\right)\right)= \\
& \quad \frac{1}{M^{0} N} \sum_{\boldsymbol{k} \sigma} P \frac{\boldsymbol{e}_{\alpha}(\boldsymbol{k}, \sigma) \boldsymbol{e}_{\beta}(\boldsymbol{k}, \sigma)}{\omega_{\boldsymbol{k} \sigma}^{2}-\omega^{2}} \\
& \quad \times \exp \left(\mathrm{i} \boldsymbol{k} \cdot\left(\boldsymbol{R}(l)-\boldsymbol{R}\left(l^{\prime}\right)\right)\right), \\
& \operatorname{Im}\left(G_{\alpha \beta}^{0}\left(l, l^{\prime}, \omega\right)\right)=-\operatorname{Im}\left(G_{\alpha \beta}^{0}\left(l, l^{\prime},-\omega\right)\right)= \\
& \quad \frac{\operatorname{sgn}(\omega)}{M^{0} N} \sum_{\boldsymbol{k} \sigma} \boldsymbol{e}_{\alpha}(\boldsymbol{k}, \sigma) \boldsymbol{e}_{\beta}(\boldsymbol{k}, \sigma) \delta\left(\omega_{\boldsymbol{k} \sigma}^{2}-\omega^{2}\right) \\
& \quad \times \exp \left(\mathrm{i} \boldsymbol{k} \cdot\left(\boldsymbol{R}(l)-\boldsymbol{R}\left(l^{\prime}\right)\right)\right) .
\end{aligned}
$$

Now, let us consider the same site ideal lattice Green function

$$
G_{\alpha \alpha}^{0}(l, l, \omega)=\frac{1}{M^{0} N} \sum_{\boldsymbol{k} \sigma} \frac{\boldsymbol{e}_{\alpha}(\boldsymbol{k}, \sigma) \boldsymbol{e}_{\alpha}(\boldsymbol{k}, \sigma)}{\omega_{\boldsymbol{k} \sigma}^{2}-(\omega+\mathrm{i} \epsilon)^{2}} .
$$

Using the orthonormality condition for eigenvectors $\boldsymbol{e}_{\alpha}(\boldsymbol{k}, \sigma)$, where $\omega>0$, one obtains

$$
\begin{gathered}
\operatorname{Im}\left(\sum_{\alpha} G_{\alpha \alpha}^{0}(l, l, \omega)\right)= \\
\frac{\pi \operatorname{sgn}(\omega)}{M^{0} N} \sum_{\boldsymbol{k} \sigma} \delta\left(\omega_{\boldsymbol{k} \sigma}^{2}-\omega^{2}\right)= \\
\frac{\pi}{M^{0} N} \sum_{\boldsymbol{k} \sigma} \frac{\delta\left(\omega_{\boldsymbol{k} \sigma}-\omega\right)}{2 \omega} .
\end{gathered}
$$

Further, when summing over all the lattice sites, one gets

$$
\begin{aligned}
& \operatorname{Im}\left(\sum_{l, \alpha} G_{\alpha \alpha}^{0}(l, l, \omega)\right)= \\
& \frac{\pi}{2 \omega M^{0}} \sum_{\boldsymbol{k} \sigma} \delta\left(\omega_{\boldsymbol{k} \sigma}-\omega\right)=\frac{\pi}{2 \omega M^{0}} Z^{0}(\omega) .
\end{aligned}
$$

Here, $Z^{0}(\omega)=\sum_{\boldsymbol{k} \sigma} \delta\left(\omega_{\boldsymbol{k} \sigma}-\omega\right)$ is the total density of states in the ideal lattice, i.e., the number of frequencies in the interval $(\omega, \omega+\mathrm{d} \omega)$. From (17), it is clear that the total density of states can be expressed as the sum of imaginary parts of the same site Green function over all the sites. Taking the obvious suggestion from this expression, we define LDS for $\omega>0$ in a non-ideal lattice by 


$$
\begin{gathered}
Z_{\alpha}(l, \omega)=\frac{2 \omega M_{l}}{\pi} \operatorname{Im}\left(G_{\alpha \alpha}(l, l, \omega)\right)= \\
\sum_{s}\left|U_{\alpha}(l, s)\right|^{2} \delta\left(\omega_{s}-\omega\right) .
\end{gathered}
$$

We note that $Z_{\alpha}(l, \omega)$ is the quantitative measure of the number of modes contributing to the vibrational behaviour of atom $l$. In the case of surfaces, $Z_{\alpha}(l, \omega)$ will in general be different for each atom and each direction in the surface region. The total density of states of the lattice can be expressed as the sum of LDS of all the atoms in the lattice

$$
\begin{aligned}
& Z(\omega)=\sum_{l, \alpha} Z_{\alpha}(l, \omega)= \\
& \frac{2 \omega}{\pi} \operatorname{Im}\left(\sum_{l, \alpha} M_{l} G_{\alpha \alpha}(l, l, \omega)\right) .
\end{aligned}
$$

In the non-ideal lattice containing point defects, surfaces or any other imperfections, the Green function can be expressed in terms of the ideal lattice Green function $G^{0}$ and perturbation caused by the presence of the imperfections. The Green function of the defect lattice is given by

$$
\left(\Phi-M \omega^{2}\right) G(\omega)=1,
$$

while that of the ideal lattice is

$$
\left(\Phi^{0}-M^{0} \omega^{2}\right) G^{0}(\omega)=1 .
$$

Assuming one defect atom, we can express (20) as

$$
\left(\Phi^{0}-M^{0} \omega^{2}+V(\omega)\right) G(\omega)=1
$$

or equivalently

$$
G(\omega)=G^{0}(\omega)-G^{0}(\omega) V(\omega) G(\omega) .
$$

In (21)-(23) the following expressions apply:

and

$$
G(\omega)=\left(\Phi-M^{0} \omega^{2}\right)^{-1}
$$

$$
\begin{gathered}
V(\omega)=\Delta \Phi-\Delta M \omega^{2}= \\
\Phi-\Phi^{0}-\left(M-M^{0}\right) \omega^{2} .
\end{gathered}
$$

The direct solution of (23) is

$$
G(\omega)=\frac{1}{1+G^{0} V} G^{0}=G^{0} \frac{1}{1+V G^{0}} .
$$

In order to obtain an equivalent expression for $G(\omega)$, one should insert (26) into the r.h.s. of (23). Then

$$
G(\omega)=G^{0}(\omega)-G^{0}(\omega) t(\omega) G^{0}(\omega)
$$

with $t(\omega)=V /\left(1+G^{0} V\right)$. Note that the Green function is given in terms of the ideal lattice Green function and the $t$ matrix. The latter is restricted to the defect space consisting of the defect and its immediate neighbours. This calculation of the defect Green function is especially suitable for vibrations of point defects and surface atoms. However, in view of difficulties with slab calculations, we used an alternative calculation of LDS.

\subsection{Thermodynamic properties}

Importantly, (19) provides local representation of the frequency spectrum. This local representation of the spectrum is particularly useful in such situations where the vibrational behaviour of a limited number of atoms is different from all other atoms in the system. For example, in a defective lattice, the vibrational behaviour of the defect and a few of its neighbours is different from that of the rest of the host atoms. In the present case, the vibrations of atoms in a few top layers on the surface are different from those of all the atoms in the interior. The use of crystal symmetry further reduces the number of atoms whose local frequency spectra must be known.

Using the local representation of the frequency spectrum, the change in the thermodynamic property is

$$
\begin{aligned}
\Delta & X(T)=\sum_{l, \alpha} \int_{0}^{\infty} \mathrm{d} \omega x(\omega, T) \\
& \times\left(Z_{\alpha}(l, \omega)-Z_{\alpha}^{0}(l, \omega)\right),
\end{aligned}
$$

where $Z_{\alpha}(l, \omega)$ is the local frequency spectrum of atom $l$ in the $\alpha$-direction in the crystal with surface while $Z_{\alpha}^{0}(l, \omega)=Z^{0}(\omega)$ is the local frequency spectrum of atom $l$ in the infinite crystal which is the same as the frequency spectrum of the ideal crystal. For an atom in the interior of both finite and infinite crystals (or the crystal with surface and the one with the cyclic boundary condition), the local spectrum is identical and therefore the surface contribution reduces to

$$
\begin{aligned}
\Delta & X(T)=\sum_{l_{s} \alpha} \int_{0}^{\infty} \mathrm{d} \omega x(\omega, T) \\
& \times\left(Z_{\alpha}\left(l_{s}, \omega\right)-Z_{\alpha}^{0}\left(l_{s}, \omega\right)\right),
\end{aligned}
$$

where $l_{s}$ is confined to the few surface layers only within the range of interatomic interactions. Within this range, the force constants are presumed to be altered due to the presence of the surface. In the case of a cubic metal, the local frequency spectra of all the atoms confined to a particular surface layer and symmetrically placed around a given surface atom are identical. Therefore, in the actual calculation, the number of atoms whose local frequency spectrum must be known is equal to the number of surface layers contained within the range of interatomic interactions. Within this range, vibrations of an atom are different from those of atoms in the interior of the crystal.

\section{Model application}

As an illustrative example, we calculate the surface contribution to low temperature lattice specific heat of tungsten due to (100) surface using LDS of atoms in the first three layers of the (100) surface. In the case of the surface contribution to lattice, the specific heat of crystals (29) transforms to

$$
\begin{aligned}
& \Delta C_{V}^{S}=k_{\mathrm{B}} \sum_{l_{s} \alpha} \int_{0}^{\infty} \mathrm{d} \omega\left(\frac{\hbar \omega}{2 k_{\mathrm{B}} T}\right)^{2} \operatorname{cosech}^{2}\left(\frac{\hbar \omega}{2 k_{\mathrm{B}} T}\right) \\
& \times\left(Z_{\alpha}\left(l_{s}, \omega\right)-Z_{\alpha}^{0}\left(l_{s}, \omega\right)\right) .
\end{aligned}
$$




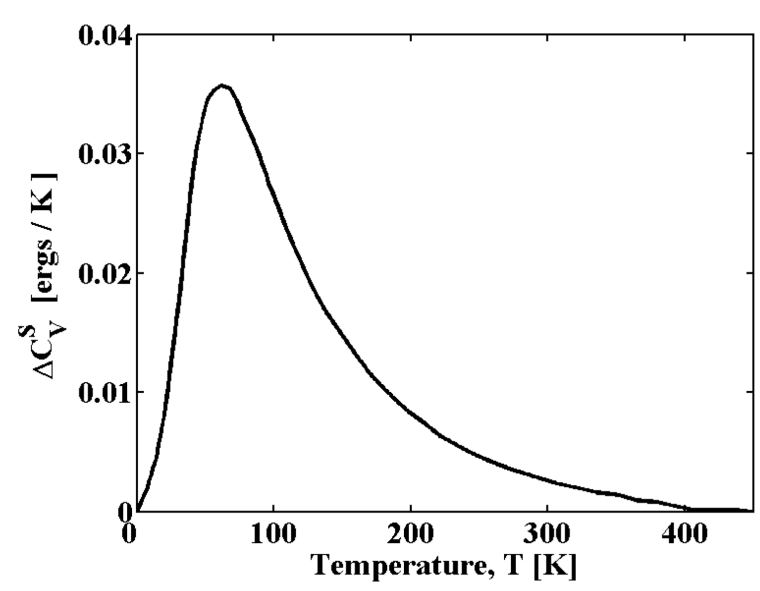

Fig. 1. Surface contribution to lattice specific heat of tungsten due to (100) surface.

In the numerical evaluation of specific heat, we have used the calculated LDS of atoms in the first three layers of the (100) surface of tungsten by Black et al. [18] who have obtained them using the recursion method. The calculated surface contribution to the lattice specific heat is plotted in Fig. 1.

We see that the surface contribution to the specific heat vanishes at $0 \mathrm{~K}$ as it must, since lattice specific heat for both the crystal with a surface and a perfect crystal vanish in the limit $T \rightarrow 0$. As the temperature increases, there is a parabolic increase in $\Delta C_{V}^{S}$ reaching a maximum and then starting to decrease, tending to zero at high temperatures. The vanishing of $\Delta C_{V}^{S}$ at high temperatures reflects the fact that in this limit each mode contributes equal amount $k_{\mathrm{B}}$ to specific heat so that both $C_{V}$ and $C_{V}^{0}$ approach $3 N k_{\mathrm{B}}$. This behaviour of $\Delta C_{V}^{S}$ is in agreement with the lattice dynamical calculations based on a slab-shaped crystal [6-8].

In the limit of low temperatures, the surface specific heat has the form (see also (1)):

$$
\Delta C_{V}^{S}=B S T^{2},
$$

where $S$ is the surface area and $B$ is a constant. Using the temperature dependence of $\Delta C_{V}^{S}$ below $35 \mathrm{~K}$ (see Fig. 1), we find $B=2 \times 10^{-5} \mathrm{erg} /\left(\mathrm{cm}^{2} \mathrm{~K}^{3}\right)$. Since, to our knowledge, there are no experimental measurements of the surface contribution to lattice specific heat of tungsten, we may compare the obtained value of $B$ with that for an isotropic elastic solid. Using (1) in conjunction with elastic constants of tungsten [33], the coefficient $B$ for an isotropic solid is found to be $2.62 \times 10^{-5} \mathrm{erg} /\left(\mathrm{cm}^{2} \mathrm{~K}^{3}\right)$. We note that the agreement is reasonable.

\section{Conclusion}

The present approach based on LDS of surface atoms provides an elegant yet straightforward method to calculate the surface contribution to the thermodynamic properties of solids.

\section{Acknowledgments}

We are grateful to Dr. J.A. Blah, Shillong, Meghalaya for his initial help in the calculation.

\section{References}

[1] A.K. Breger, A.A. Zhukovitskii, Acta Physiochim. USSR 21, 1001 (1946).

[2] E.W. Montroll, J. Chem. Phys. 18, 183 (1950).

[3] M. Dupuis, R.M. Mazo, L. Onsager, J. Chem. Phys. 33, 1452 (1960).

[4] A.A. Maradudin, E.W. Montroll, G.H. Weiss, I.P. Ipatova, Theory of Lattice Dynamics in the Harmonic Approximation, Academic, New York 1971, Ch. IX, p. 4.

[5] R.F.S. Hearmon, An Introduction to Applied Anisotropic Elasticity, Oxford University Press, London 1961, p. 72.

[6] R.E. Allen, F.W. Dewette, J. Chem. Phys. 51, 4820 (1969).

[7] T.S. Chen, G.P. Alldredge, F.W. Dewette, R.E. Allen, J. Chem. Phys. 55, 3121 (1971).

[8] A.A. Maradudin, R.F. Wallis, L. Dobrzynski, Surface, Phonons and Polaritons, Handbook of Surfaces and Interfaces, Vol. III, Ed. L. Dobrzynski, Gariland STPM Press, New York 1980.

[9] K. Portz, A.A. Maradudin, Phys. Rev. B 16, 3535 (1977).

[10] P.N. Ram, Radiat. Eff. Defects Solids 118, 1 (1991).

[11] D. Kalkstein, P. Soven, Surf. Sci. 26, 85 (1971).

[12] M. Mostoller, T. Kaplan, Phys. Rev. B 19, 552 (1979).

[13] M. Mostoller, U. Landman, Phys. Rev. B 20, 1755 (1979).

[14] D. Castiel, L. Dobrzynski, D. Spanjaard, Surf. Sci. 59, 252 (1976).

[15] P.D. Ditlevsen, J.K. Norskov, Surf. Sci. 254, 261 (1991).

[16] E. Hulpke, M. Huppauff, D.M. Smilgies, A.D. Kulkarni, F.W. de Wette, Phys. Rev. $B$ 45, 1820 (1992).

[17] R. Haydock, V. Heine, M.J. Kelly, J. Phys. C 5, 2845 (1972).

[18] J.E. Black, B. Laks, D.L. Mills, Phys. Rev. B 22, 1818 (1980).

[19] K. Masuda, Z. Nat.forsch. 36a, 454 (1981).

[20] K. Masuda, Lettere Nuovo Cim. 31, 393 (1981). 
[21] G. Treglia, M.C. Deszonqueres, J. Phys. (France) 46, 987(1985).

[22] N. Luo, W. Xu, S. Shen, Phys. Status Solidi $B$ 147, 511 (1988).

[23] Z. Tang, N.R. Aluru, Phys. Rev. B 74, 235441 (2006).

[24] P.H. Dederichs, R. Zeller, In Point Defects in Metals II, Vol. 87 of Springer Tracts in Modern Physics, Eds. G. Hohler, E. Niekisch, Springer, Berlin 1979.

[25] R. Zeller, P.H. Dederichs, Z. Phys. B 25, 139 (1976).

[26] P.N. Ram, Phys. Rev. B 43, 6977 (1991).

[27] J.A. Blah, S.S. Pohlong, P.N. Ram, Phys. Rev. B 49, 15055 (1994).
[28] P.N. Ram, J. Phys. C Solid State Phys. 21, 25 (1988).

[29] B.K. Rath, P.N. Ram, Phys. Status Solidi B 156, 137 (1989).

[30] P.N. Ram, V. Gairola, P.D. Semalty, J. Phys. Chem. Solids 94, 41 (2016).

[31] V. Gairola, P.D. Semalty, P.N. Ram, Pramana J. Phys. 80, 1041 (2013).

[32] A. Iskandar, A. Gwiazda, Y. Huang, M. Kazan, A. Bruyant, M. Jabbal, G. Lerondel, J. Appl. Phys. 120, 095106(2016).

[33] C. Kittel, Introduction to Solid State Physics, 4th ed., Wiley, New York 1971, Ch. 4. 